\title{
IBRD Operational Decision Framework
}

\section{October 1, 2009}

\author{
Primary Authors: \\ Robert Greenwalt, Wilthea Hibbard, Ellen Raber, Tina Carlsen, Karen Folks, Don \\ MacQueen, Sav Mancieri, Thomas Bunt, John Richards, and Joy Hirabayashi-Dethier \\ Lawrence Livermore National Laboratory (LLNL) \\ with contributions from: \\ Mark D. Tucker, Lynn Yang, and Robert Knowlton \\ Sandia National Laboratories (SNL)
}




\section{Disclaimer}

This document was prepared as an account of work sponsored by an agency of the United States government. Neither the United States government, Lawrence Livermore National Security, LLC, nor any of their employees makes any warranty, expressed or implied, or assumes any legal liability or responsibility for the accuracy, completeness, or usefulness of any information, apparatus, product, or process disclosed, or represents that its use would not infringe privately owned rights. Reference herein to any specific commercial product, process, or service by trade name, trademark, manufacturer, or otherwise does not necessarily constitute or imply its endorsement, recommendation, or favoring by the United States government or Lawrence Livermore National Security, LLC. The views and opinions of authors expressed herein do not necessarily state or reflect those of the United States government or Lawrence Livermore National Security, LLC, and shall not be used for advertising or product endorsement purposes.

\section{Auspices Statement}

This work performed under the auspices of the U.S. Department of Energy by Lawrence Livermore National Laboratory under Contract DE-AC52-07NA27344. 
The IBRD Operational Decision Framework in this document is an expansion of an emerging general risk management framework under development by an interagency working group. It provides the level of detail necessary to develop a general Consequence Management Guidance Document for biological contamination remediation and restoration. It is the intent of this document to support both wide area and individual site remediation and restoration activities.

This product was initiated as a portion of the IBRD Task 1 Systems Analysis to aid in identification of wide area remediation and restoration shortcomings and gaps. The draft interagency general risk management framework was used as the basis for the analysis. The initial Task 1 analysis document expanded the draft interagency framework to a higher level of resolution, building on both the logic structure and the accompanying text explanations. It was then employed in a qualitative manner to identify responsible agencies, data requirements, tool requirements, and current capabilities for each decision and task. This resulted in identifying shortcomings and gaps needing resolution. Several meetings of a joint LLNL/SNL working group reviewed and approved the initial content of this analysis.

At the conclusion of Task 1, work continued on the expanded framework to generate this Operational Decision Framework which is consistent with the existing interagency general risk management framework. A large LLNL task group met repeatedly over a three-month period to develop the expanded framework, coordinate the framework with the biological remediation checklist, and synchronize the logic with the Consequence Management Plan table of contents.

The expanded framework was briefed at a large table top exercise reviewing the interagency risk management framework. This exercise had representation from major US metropolitan areas as well as national agencies. This product received positive comments from the participants.

Upon completion of the Operational Decision Framework, another joint LLNL/SNL working group conducted a day-long review. Identified modifications were made to the document, resulting in the included product.

Special thanks are given to the extensive contributions of the LLNL author team, as well as to the technical review and comments from the SNL contributors. 


\section{Operational Phases for Response and Consequence Management Framework}

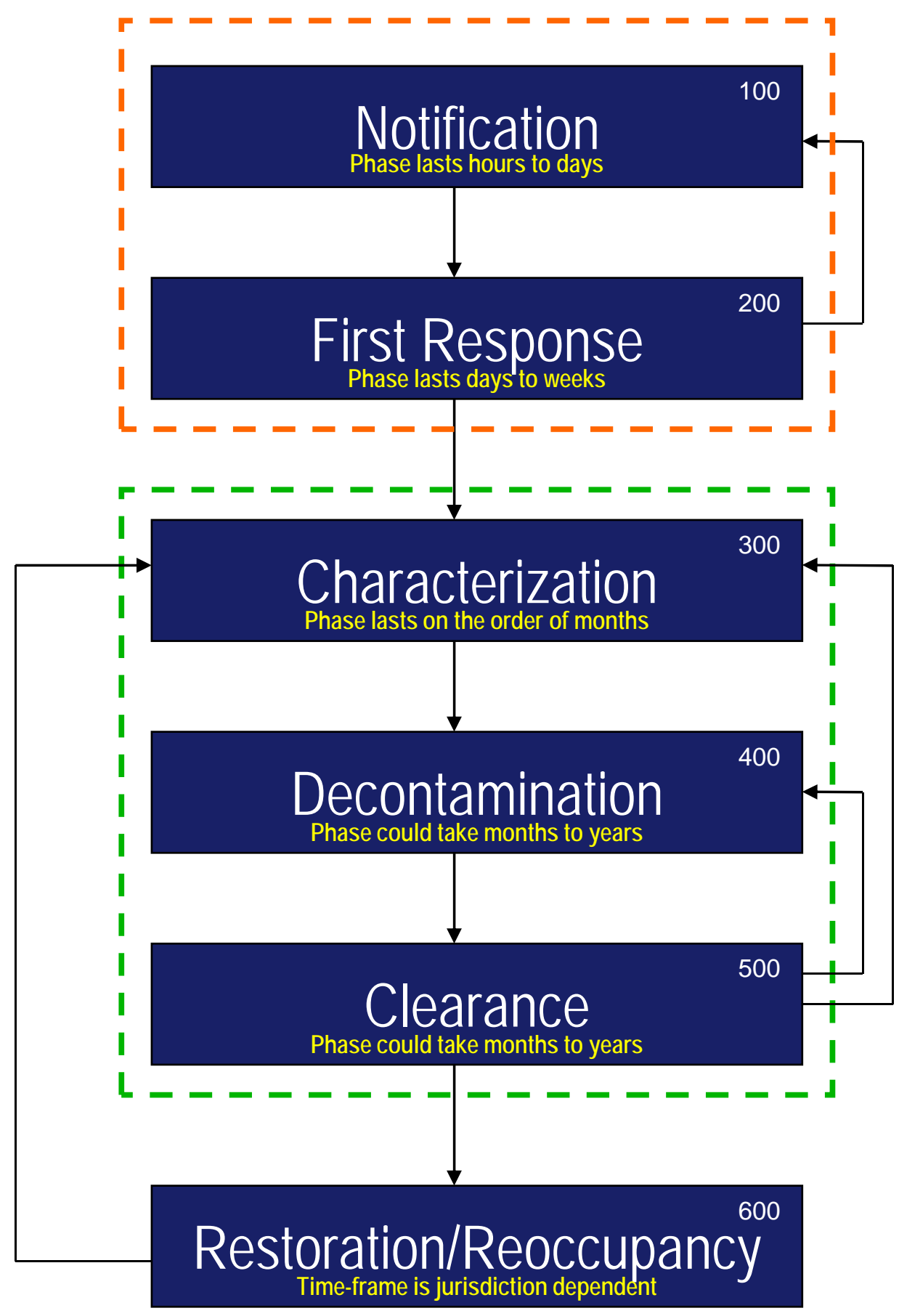

[Response]

[Remediation]

Steps can proceed in parallel,

are iterative and multiple

areas or facilities can be addressed

independently or simultaneously 


\section{Biological Agent Incident-Response Decision Process (1 of 5)}

\section{NOTIFICATION}
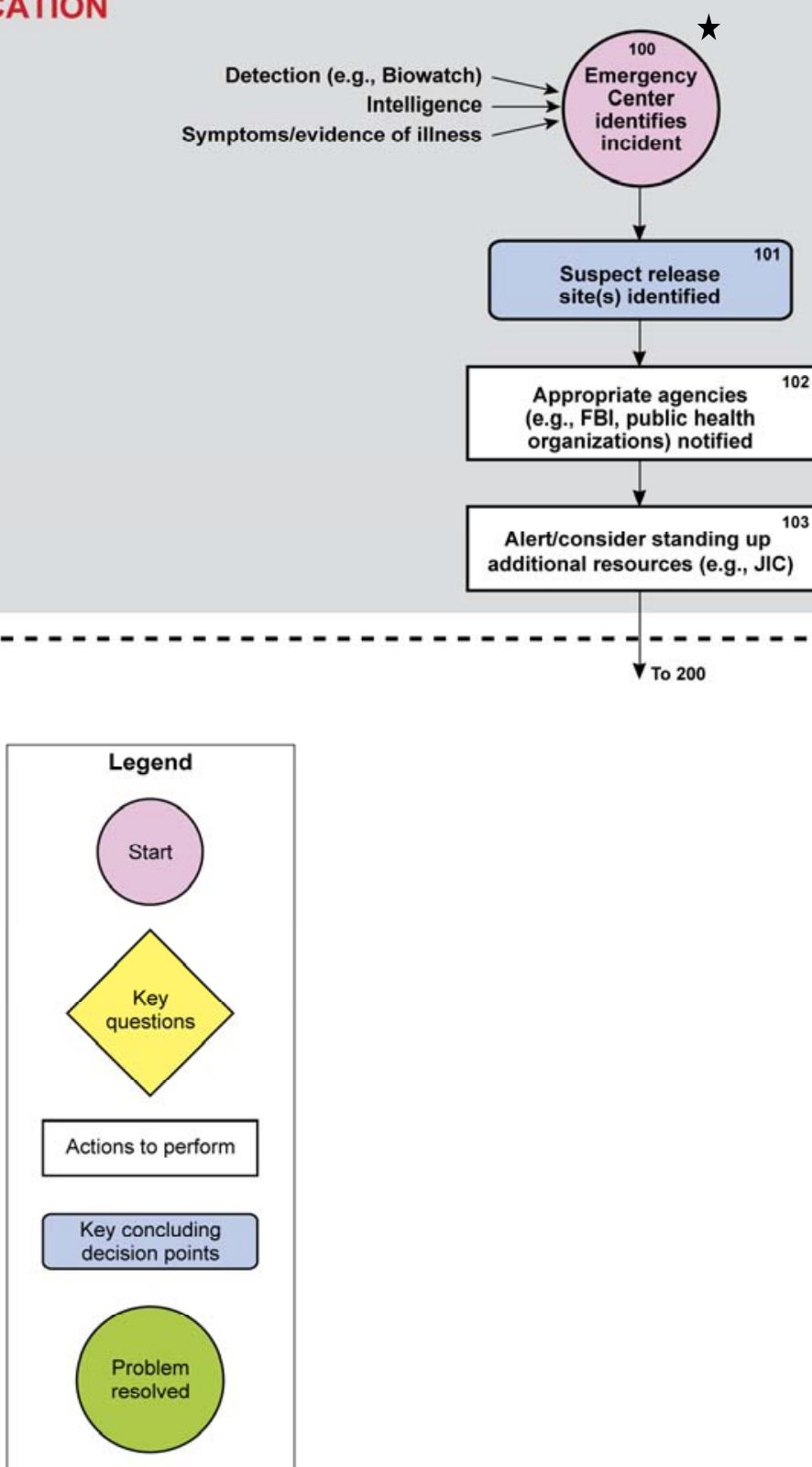


\section{Biological Agent Incident-Response Decision Process (2 of 5) \\ From 103}

\section{FIRST RESPONSE}

204

Control site access, contain area, identify and prioritize contaminated/affected areas and operations

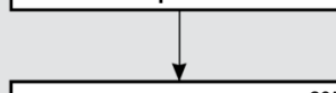

Perform additional emergency actions according to specific

Hazmat response plan, e.g.:

- Decon with soap \& water

- Evacuation/containment

- Operational/utility

shutdowns

- Public health options

- Notifications

- Screening sampling

- Shelter in place
205

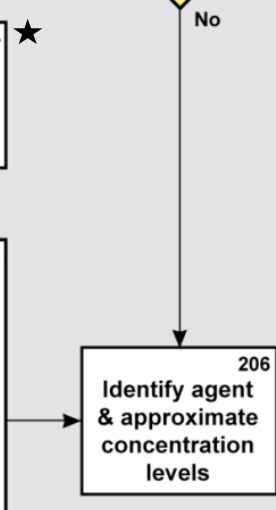

Conduct initial threat assessment, and perform:
- General hazard analysis/site safety
- Preliminary Hazmat response
- Initial control measures
- Rapid intelligence/data gathering
- Risk communication strategy

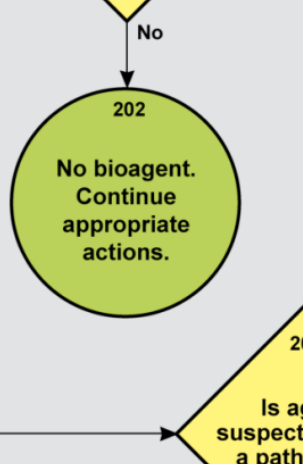

Is bioagent
suspected?
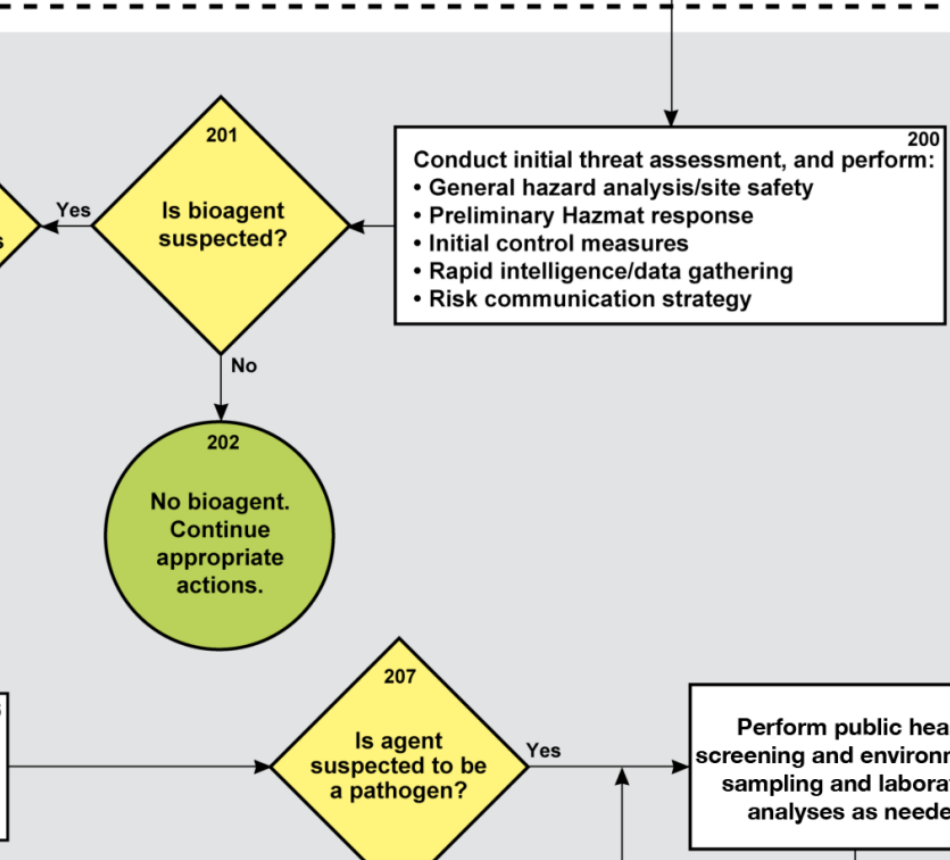

Is agent

208

Perform public health screening and environmental sampling and laboratory analyses as needed
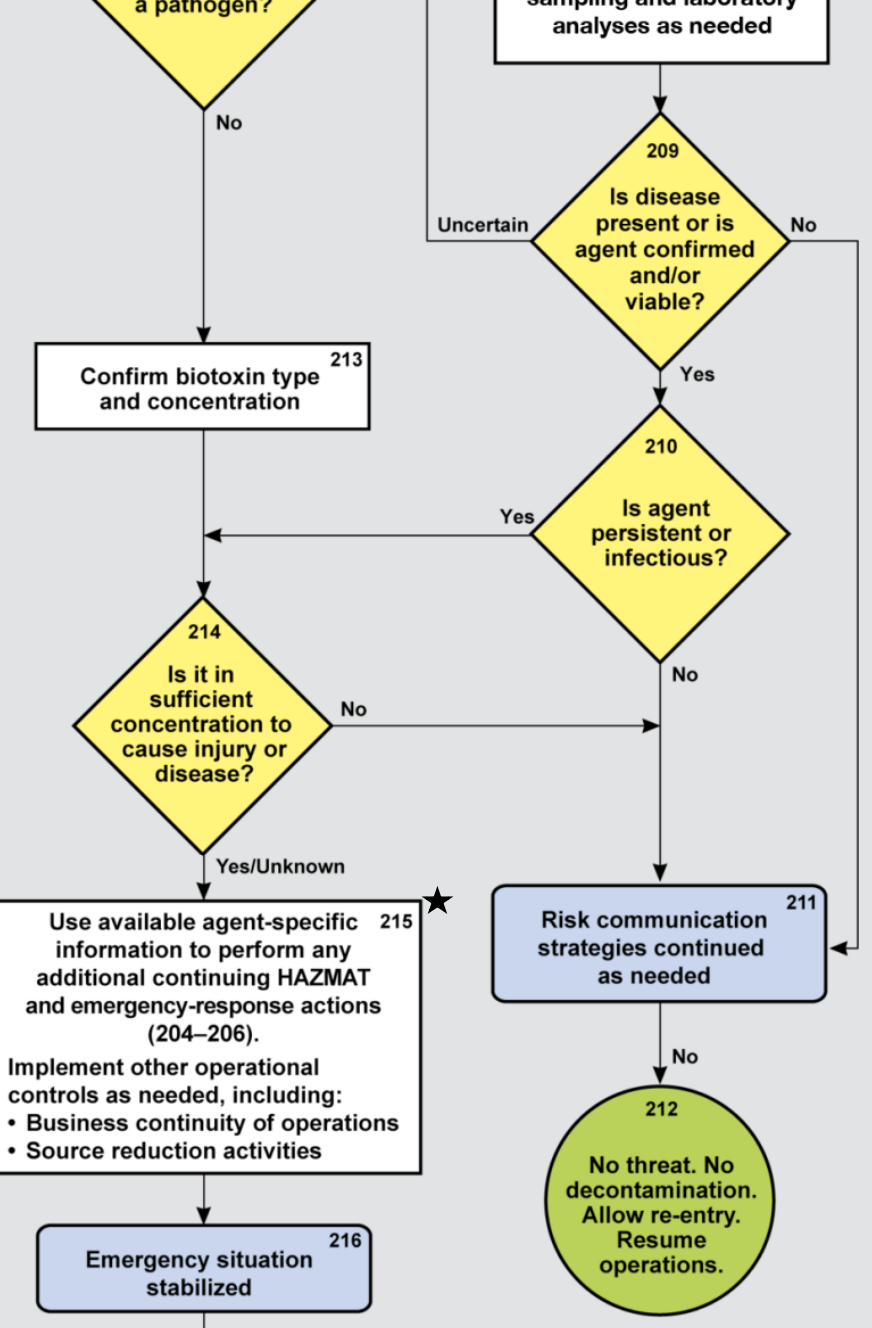


\section{Biological Agent Incident-Response Decision Process (3 of 5)}

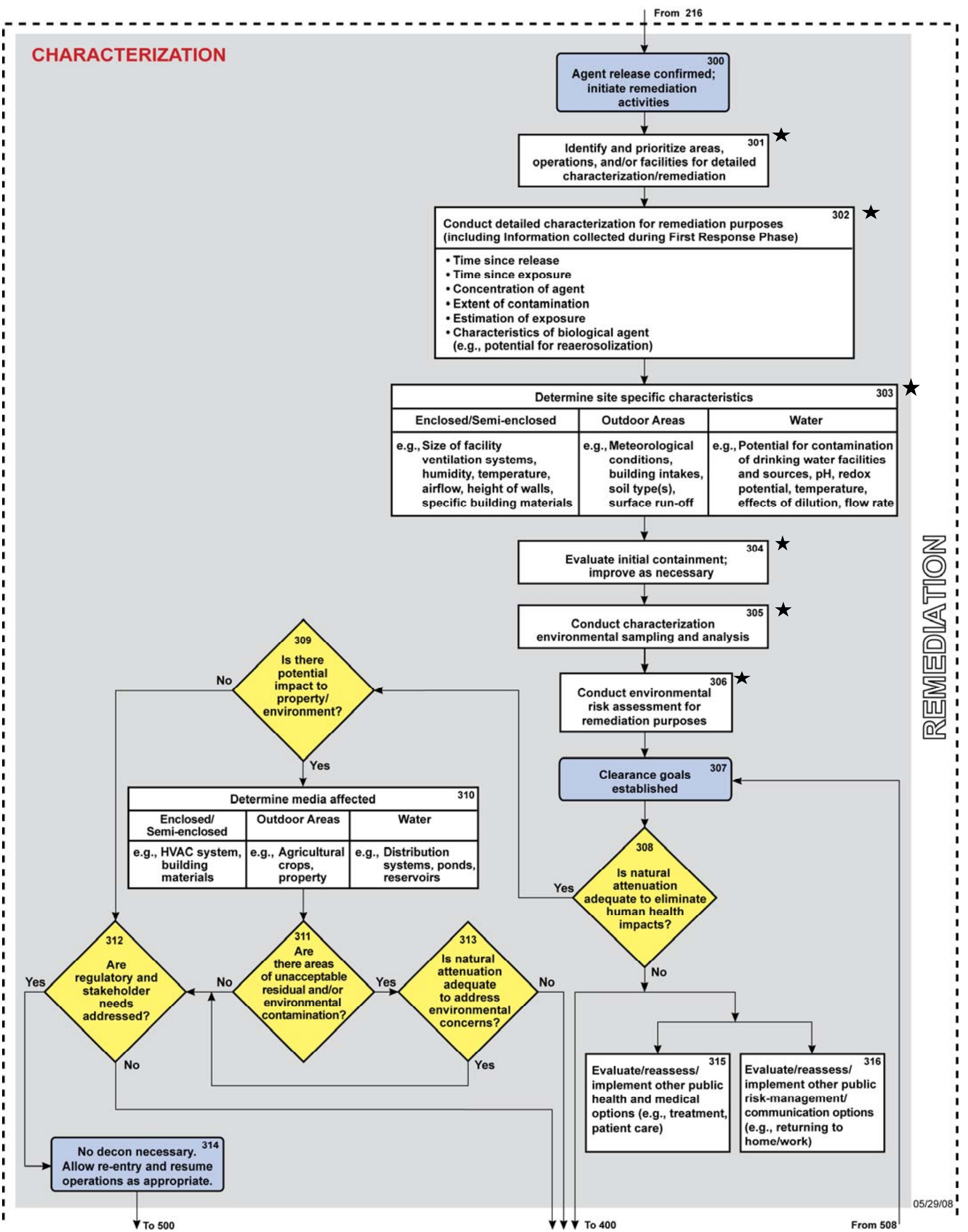




\section{Biological Agent Incident-Response Decision Process (4 of 5)}

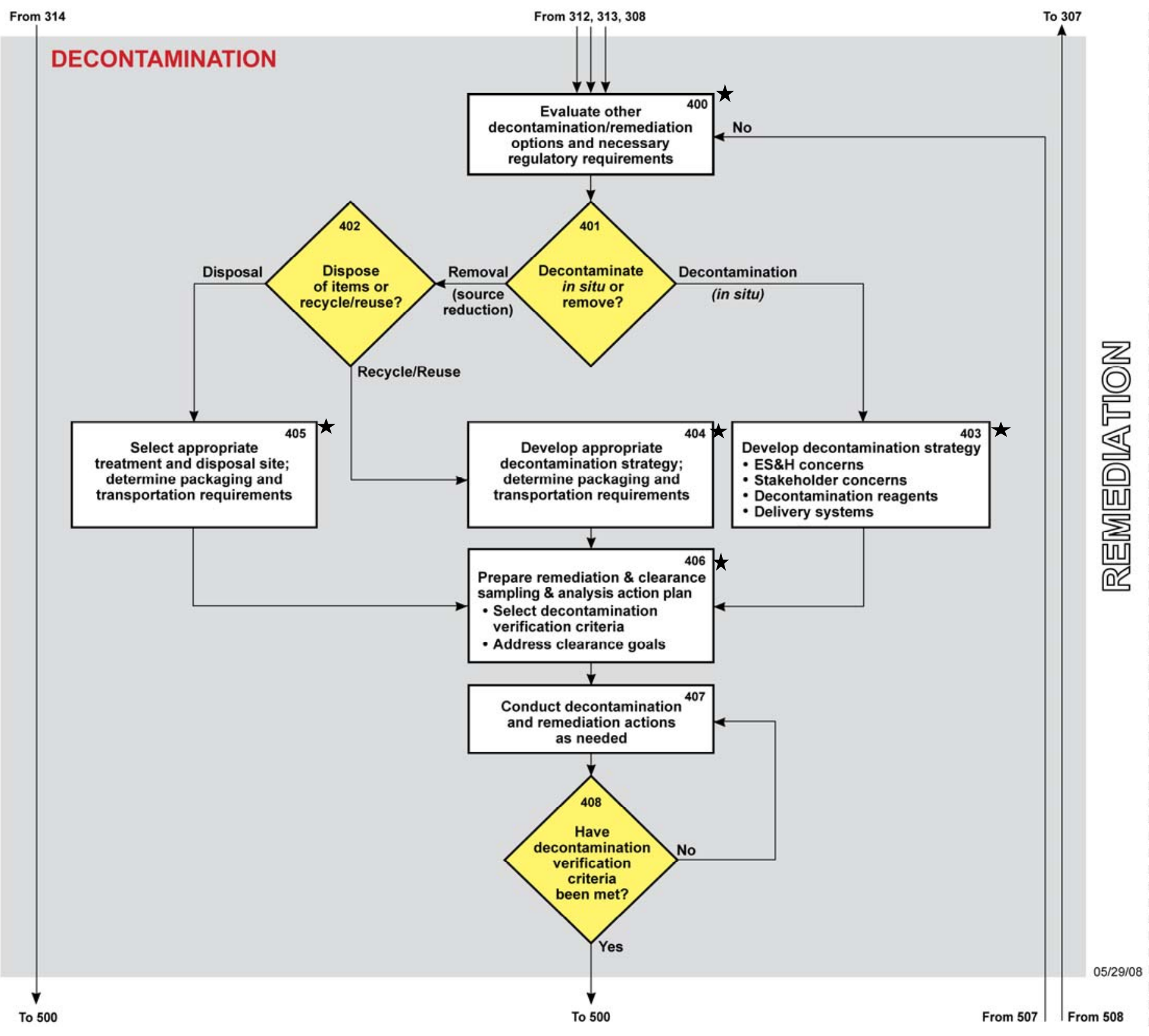




\section{Biological Agent Incident-Response Decision Process (5 of 5)}
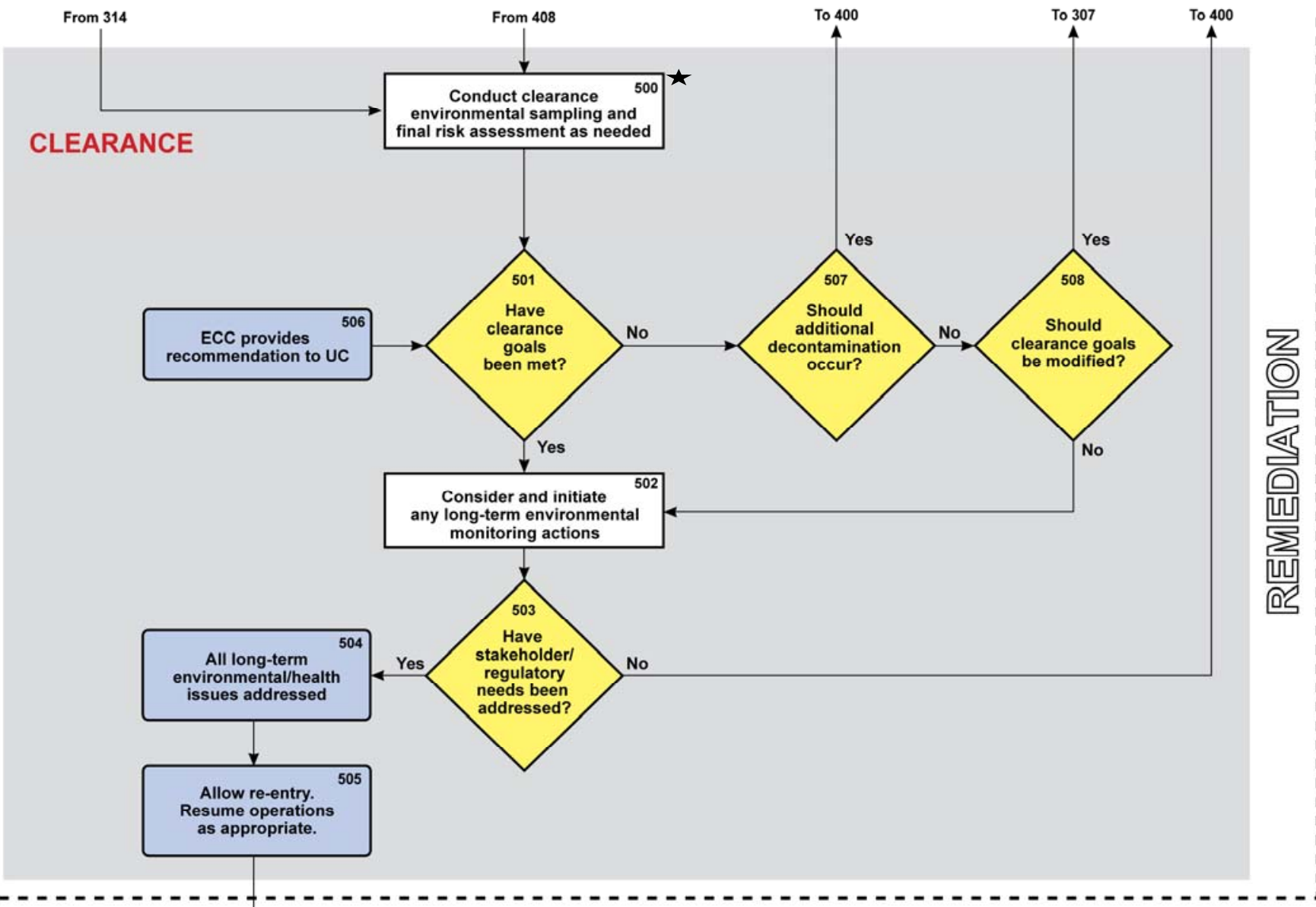

\section{REOCCUPANCY}
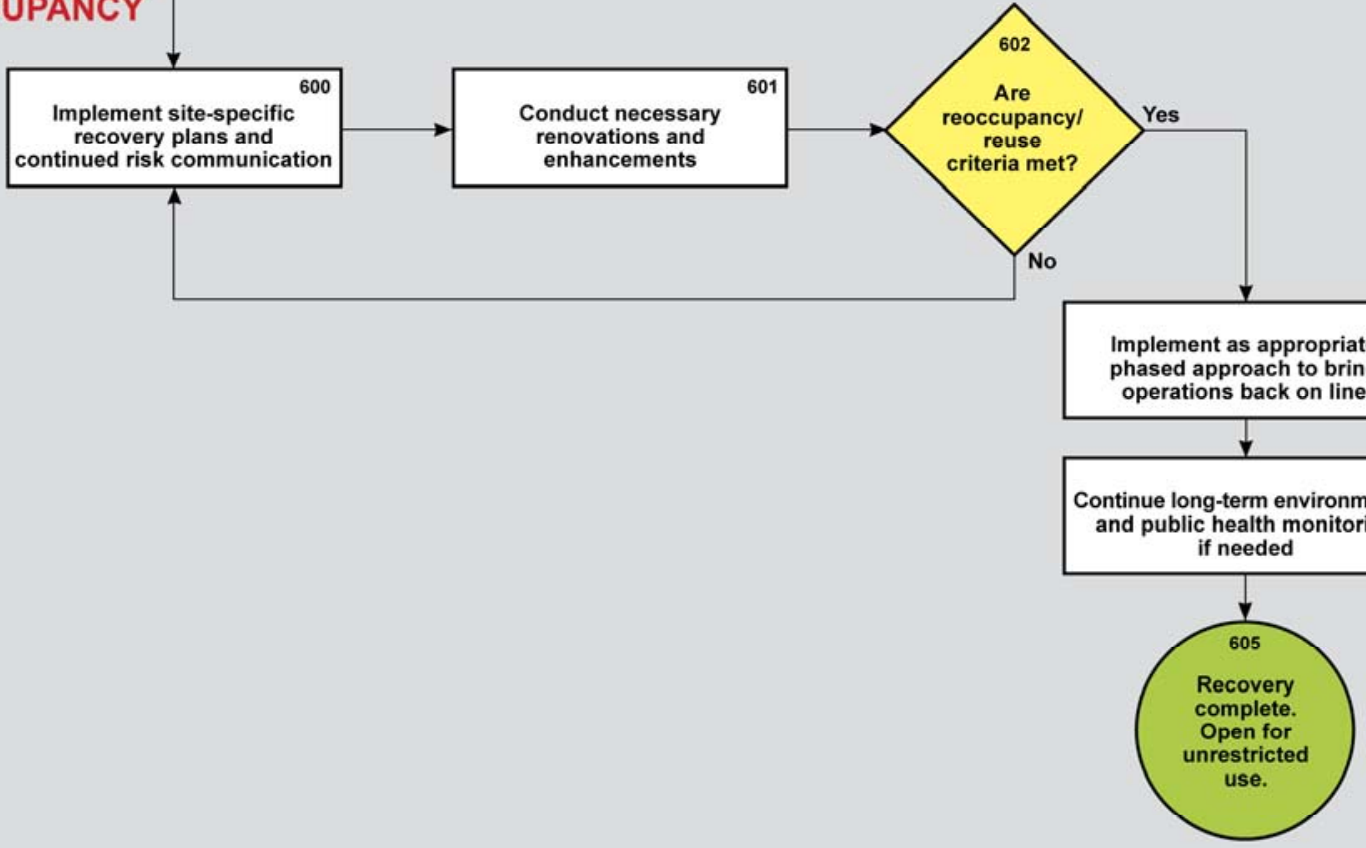

phased approach to bring operations back on line monitoring 
NOTIFICATION

(100 Expanded)
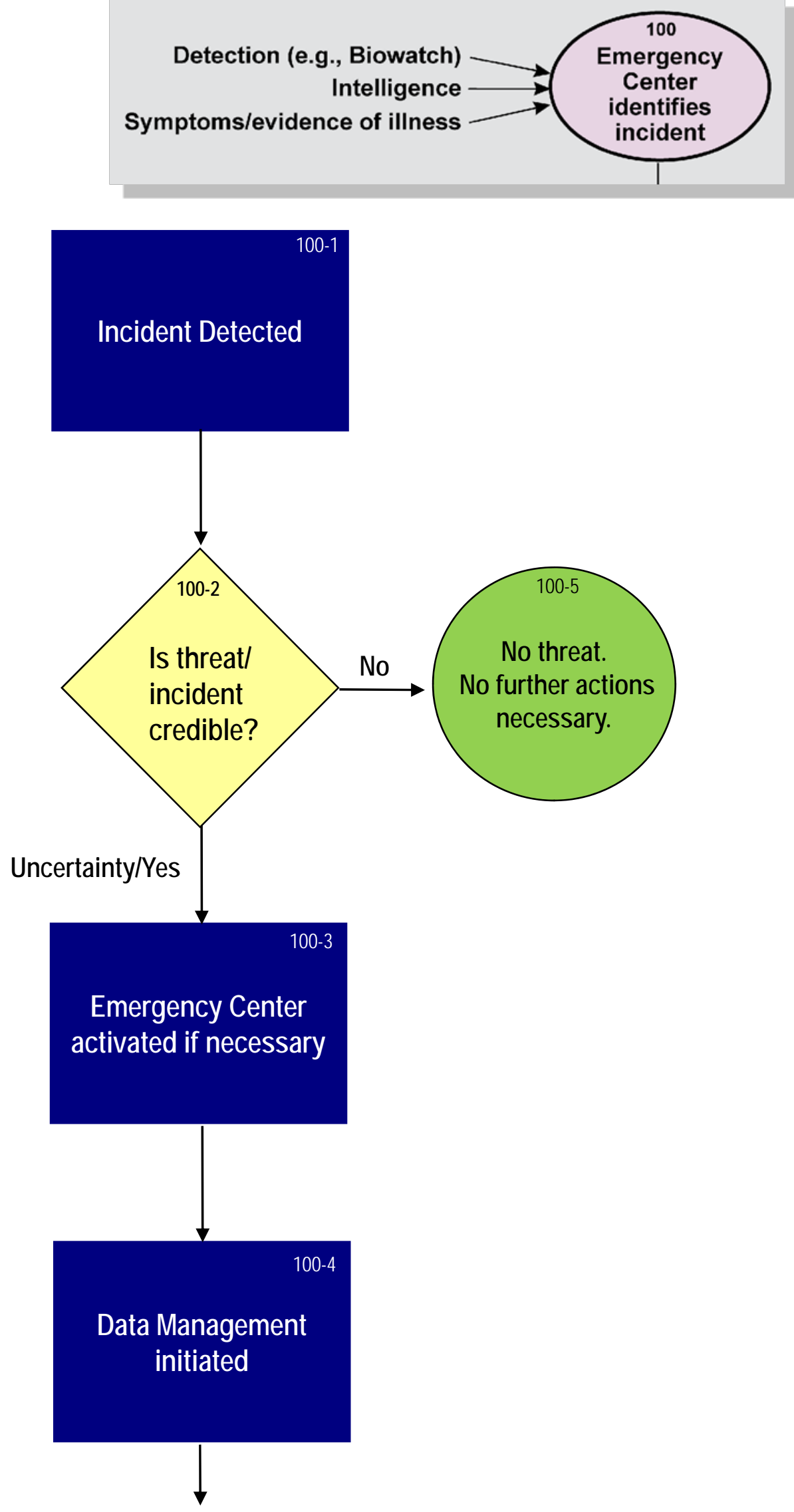


\section{FIRST RESPONSE (204 Expanded)}

Control site access, contain area, identify and prioritize contaminated/affected areas and operations

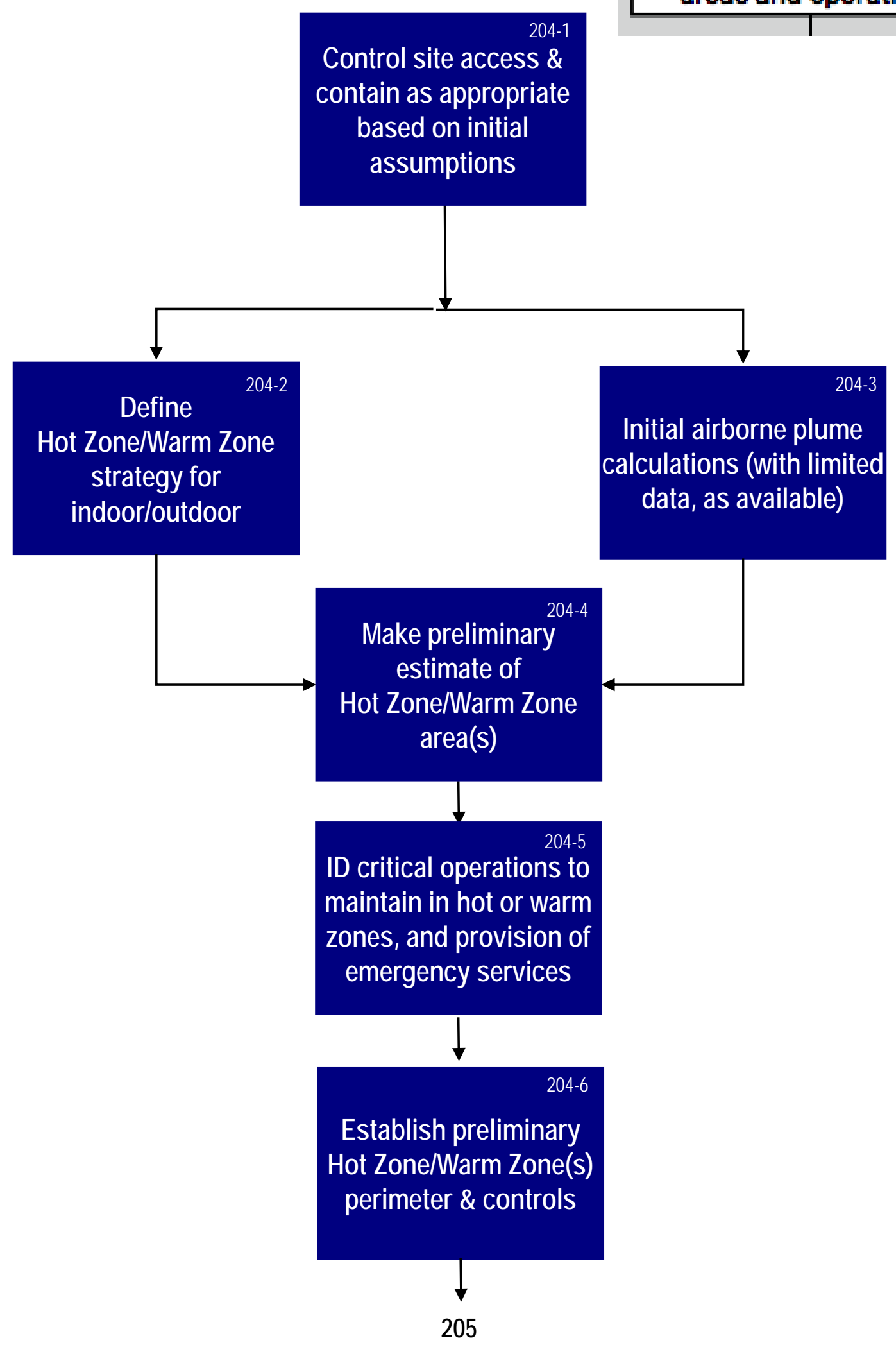




\section{FIRST RESPONSE (208 Expanded)}

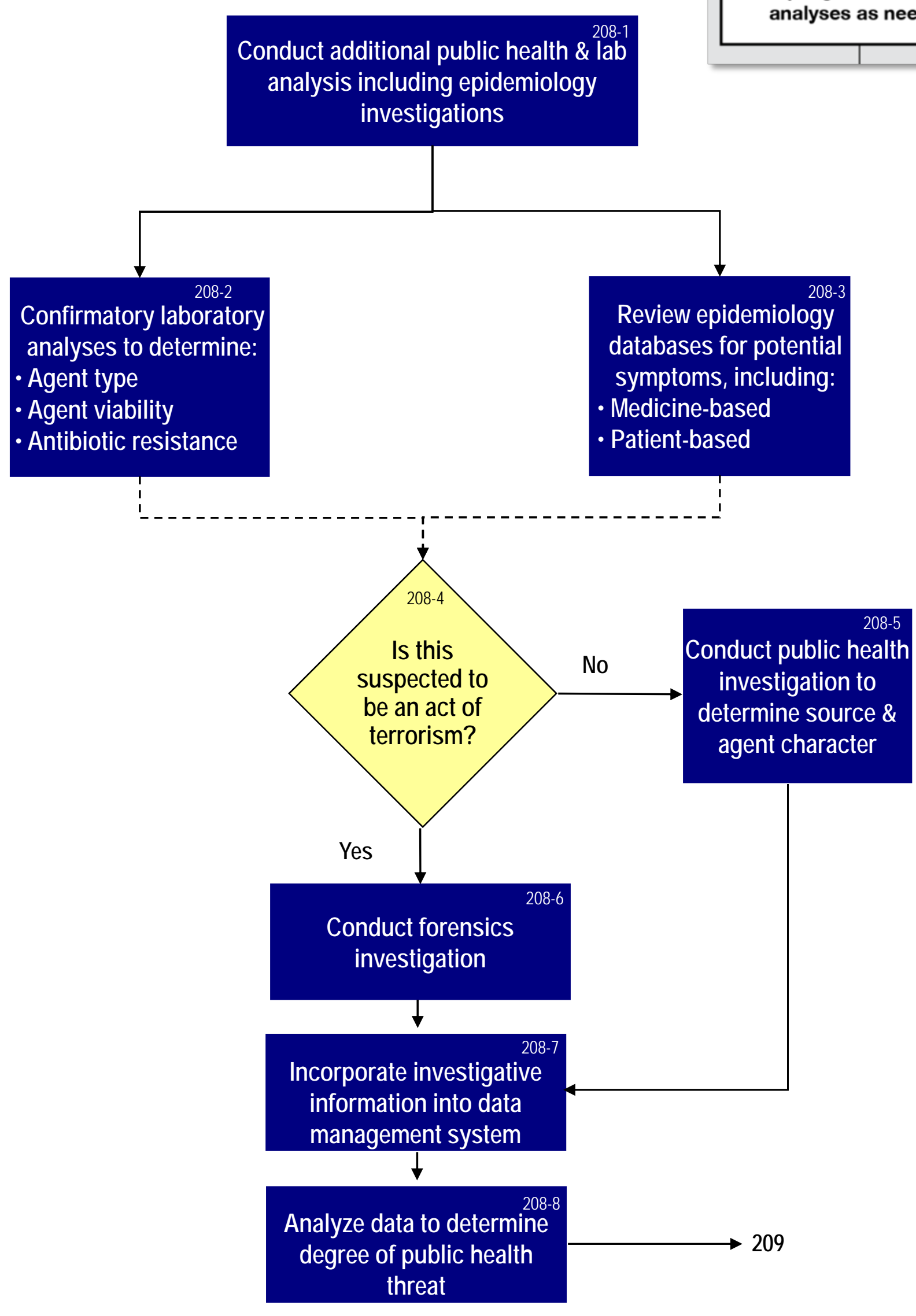

Perform public health screening and environmental sampling and laboratory analyses as needed

\section{analysis including epidemiology \\ investigations}

Confirmatory laboratory

Agent type

- Agent viability

- Antibiotic resistance 


\section{FIRST RESPONSE (215 Expanded)}

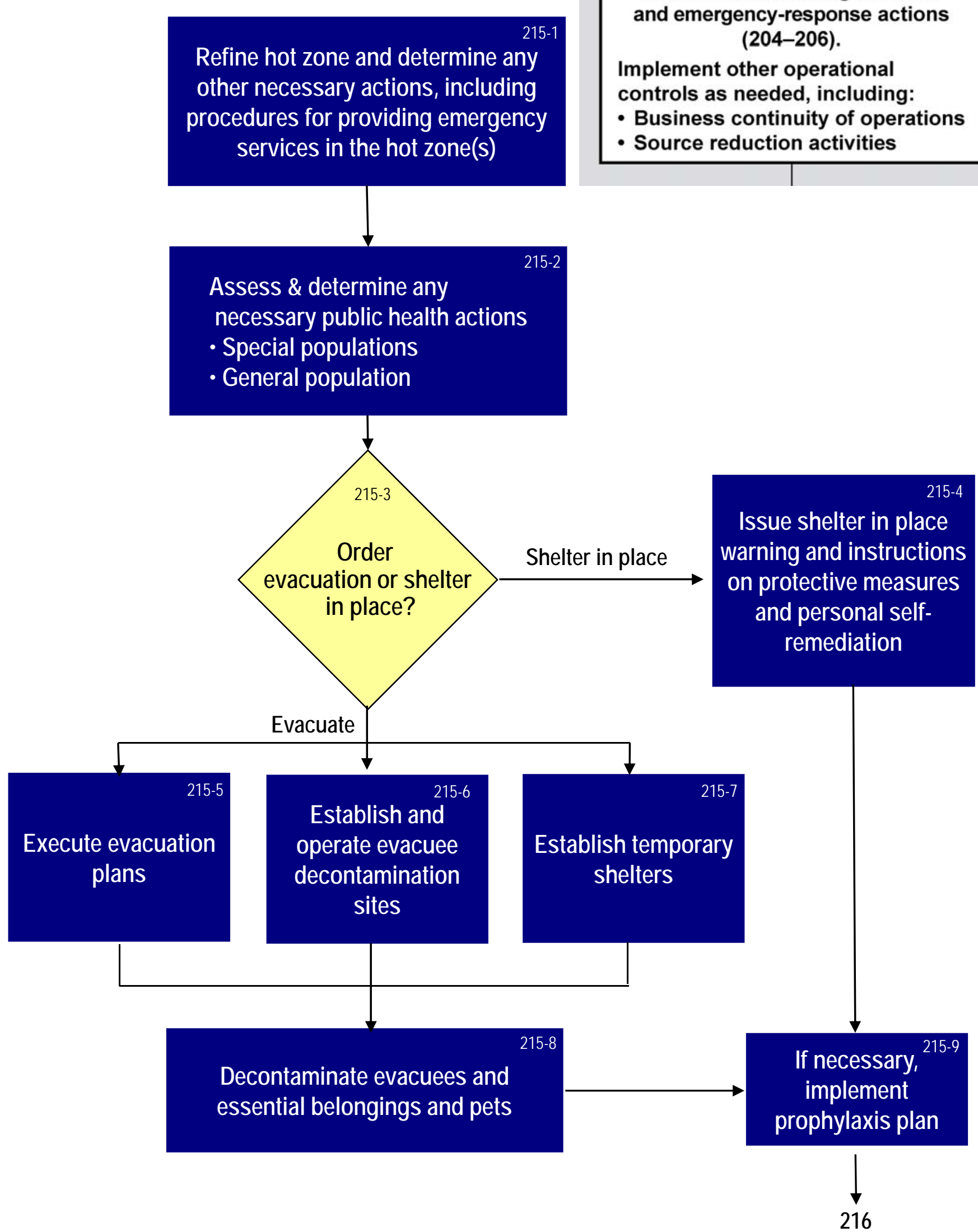

Use available agent-specific 215 information to perform any additional continuing HAZMAT and emergency-response actions (204-206).

Implement other operational controls as needed, including:

- Business continuity of operations

- Source reduction activities 


\section{CHARACTERIZATION (301 expanded)}

Identify and prioritize areas,

operations, and/or facilities for detailed characterization/remediation

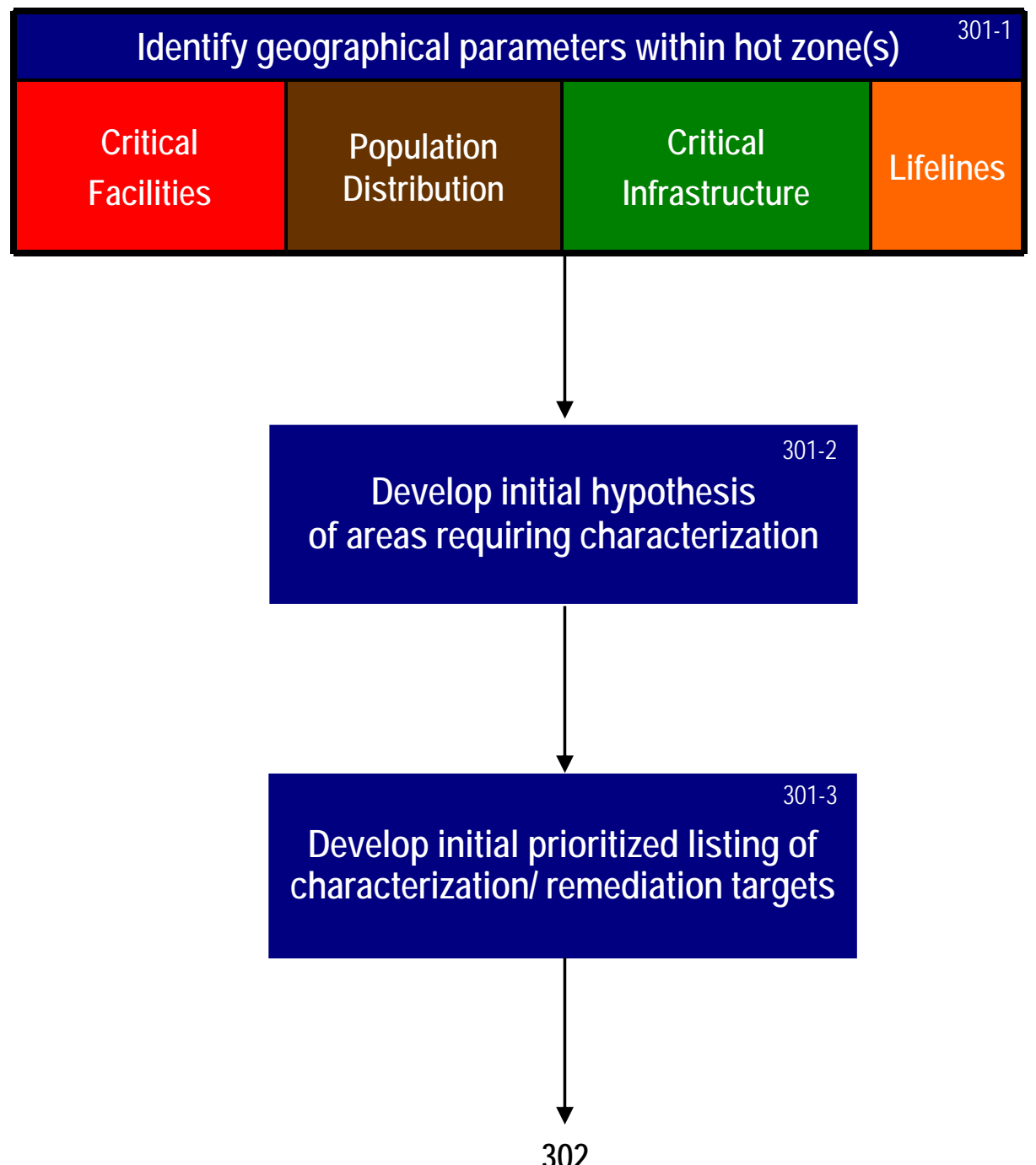

302 


\section{CHARACTERIZATION (302 expanded)}

- Time since release

- Time since exposure

- Concentration of agent

- Extent of contamination

- Estimation of exposure

- Characteristics of biological agent

(e.g., potential for reaerosolization)

\section{$302-1$}

Gather/collect information from

first response (e.g., time since release, concentration maps, characteristics of agent)

As task 303

progresses, continually reassess modeling results
Interpret and compare with contamination models verify models with response sample data

- Atmospheric transport models (IMAAC)

- Indoor airflow models

- Water flow models

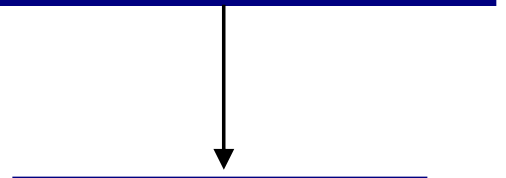

302-4

Identify data gaps

necessitating additional

characterization activities 


\section{CHARACTERIZATION (303 expanded)}

\begin{tabular}{|c|c|c|}
\hline \multicolumn{3}{|c|}{ Determine site specific characteristics } \\
\hline \multicolumn{1}{|c|}{ Enclosed/Semi-enclosed } & Outdoor Areas & Water \\
\hline $\begin{array}{c}\text { e.g., Size of facility } \\
\text { ventilation systems, } \\
\text { humidity, temperature, } \\
\text { airflow, height of walls, } \\
\text { specific building materials }\end{array}$ & $\begin{array}{c}\text { e.g., Meteorological } \\
\text { conditions, } \\
\text { building intakes, } \\
\text { soil type(s), } \\
\text { surface run-off }\end{array}$ & $\begin{array}{c}\text { e.g., Potential for contamination } \\
\text { of drinking water facilities } \\
\text { and sources, pH, redox } \\
\text { potential, temperature, } \\
\text { effects of dilution, flow rate }\end{array}$ \\
\hline
\end{tabular}

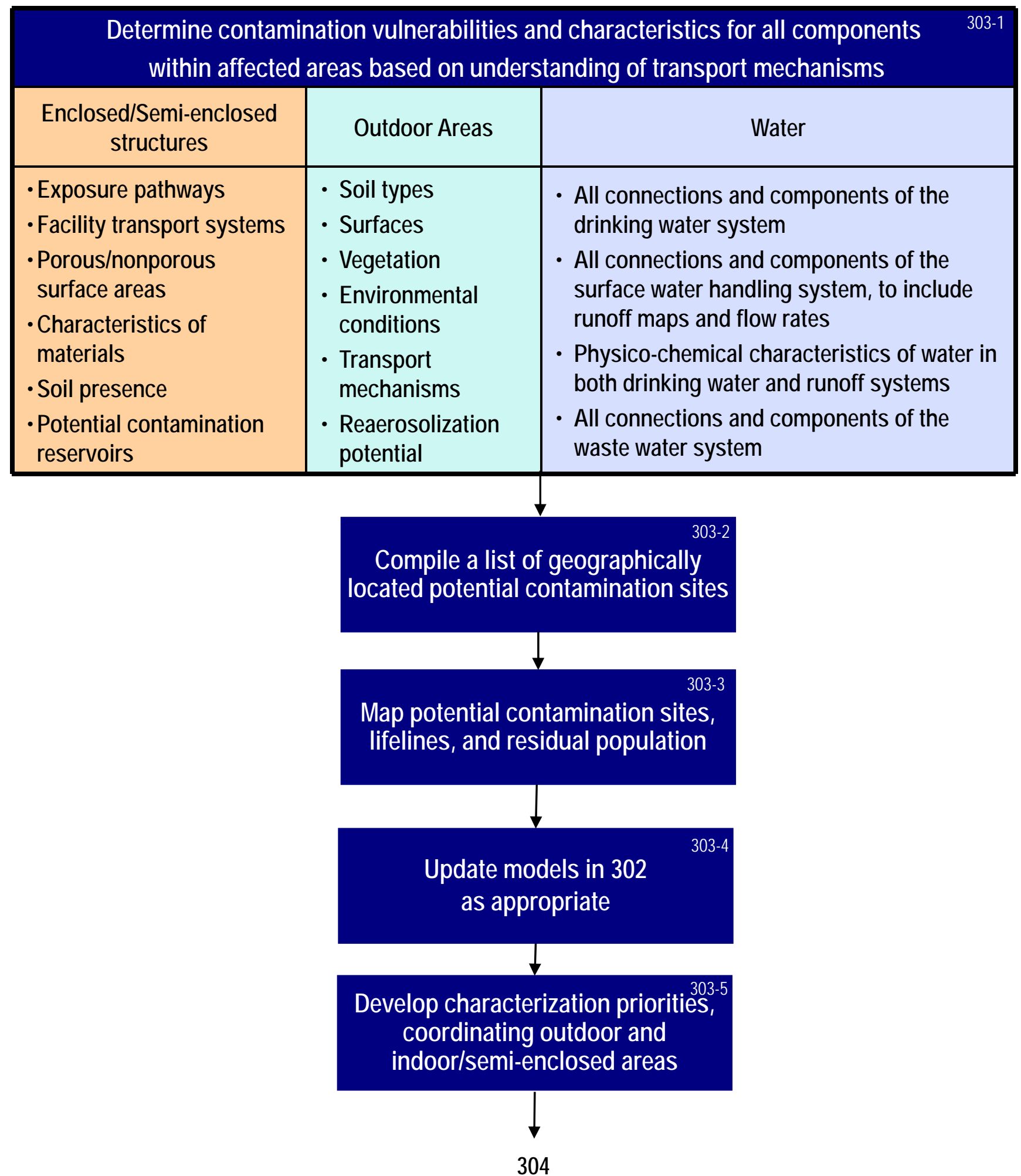




\section{CHARACTERIZATION (304 expanded)}

Evaluate initial containment; improve as necessary

304-1

Update hot zone(s)

boundaries

304-2

Adjust warm zone(s)

boundaries

304-3

Adjust boundary controls

as necessary

Seal buildings \& HVAC systems within hot zone(s)
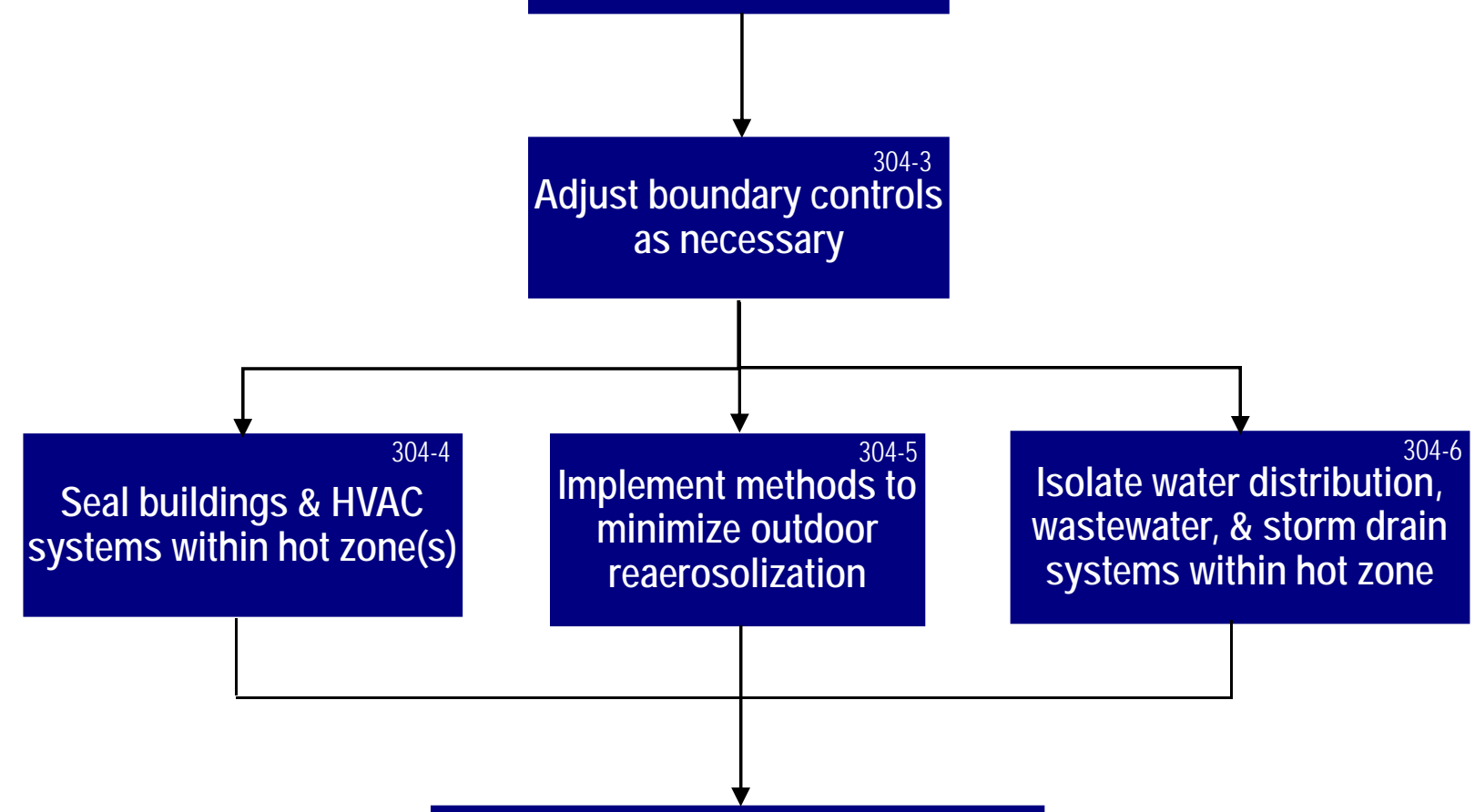

Continue to reassess hot and warm zone boundaries as characterization and clearance progress 


\section{CHARACTERIZATION (305 expanded)}

Conduct characterization environmental sampling and analysis

Develop comprehensive characterization sampling strategies

- Consider a phased approach to rapidly refine the limits of contamination

- Develop data quality objectives

- Consider statistical and judgmental criteria

- Employ iterative modeling approach to optimize sampling

- Establish minimum characterization sampling requirements

- Consider employing a zonal approach for sampling

- Consider surface, air, and forced air sampling

- Address all necessary information requirements for risk assessment (including inhalation, cutaneous or gastrointestinal risks)

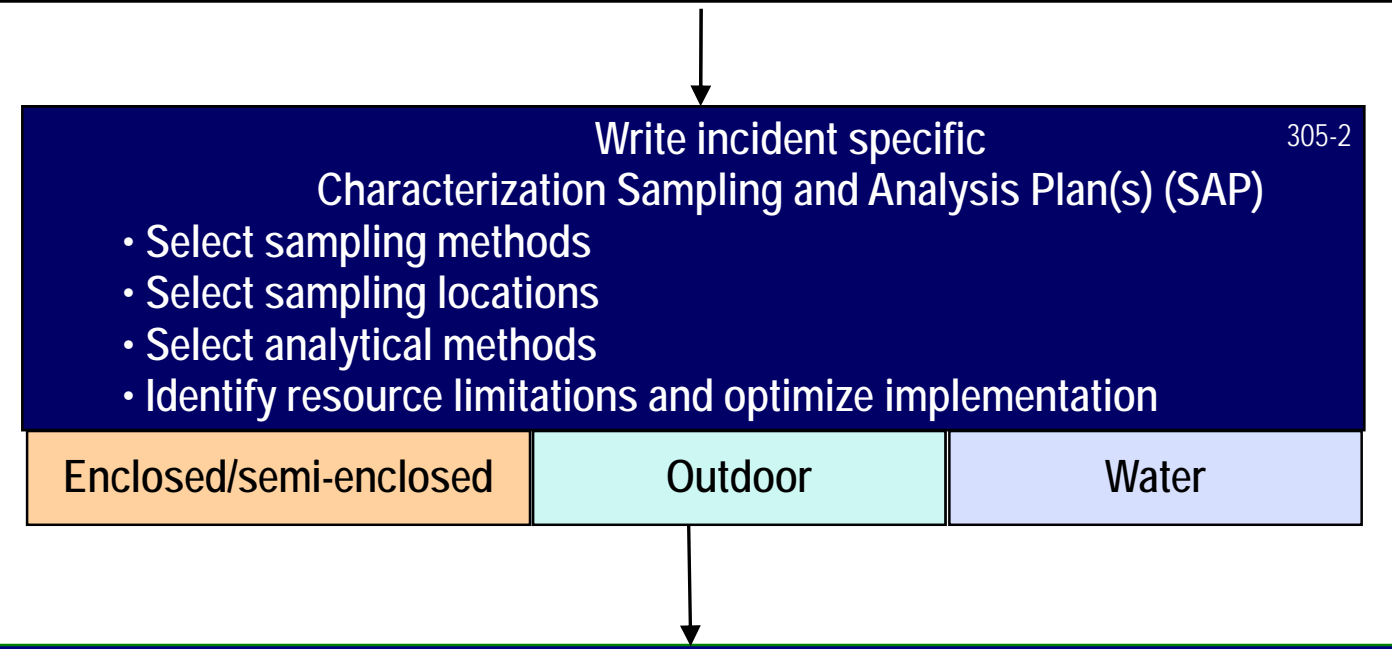

Conduct characterization sampling and analysis

Enclosed/semi-enclosed

Outdoor

Water

Re-evaluate and adjust sampling strategy, plans, and methods

as necessary 
Conduct technical risk assessment including consideration of the "no action"/natural attenuation alternative

Evaluate \& incorporate socio-economic implications

Use risk management process to set clearance goals 


\section{DECONTAMINATION (expanded 400)}

Evaluate other decontamination/remediation options and necessary regulatory requirements

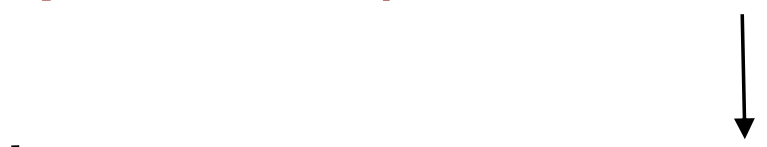

$400-1$

Evaluate decontamination options for media affected, considering for example: Volume, Extent of contamination, Availability of resources, Accessibility, Weather

\begin{tabular}{|l|l|l|l|}
\hline \multicolumn{1}{|c|}{$\begin{array}{c}\text { Enclosedl } \\
\text { Semi-enclosed }\end{array}$} & \multicolumn{2}{|c|}{ Outdoor Areas } & \multicolumn{1}{c|}{ Water } \\
\hline $\begin{array}{l}\text { e.g., HVAC system, building } \\
\text { materials, fixed \& moveable } \\
\text { property, sensitive } \\
\text { equipment, rolling stock }\end{array}$ & $\begin{array}{l}\text { Natural } \\
\text { e.g., soil, } \\
\text { vegetation, } \\
\text { agricultural } \\
\text { crops, } \\
\text { livestock }\end{array}$ & $\begin{array}{l}\text { Man-made } \\
\text { e.g., building } \\
\text { exteriors, } \\
\text { pavement } \\
\text { structures, fixed } \\
\text { and moveable } \\
\text { property, } \\
\text { sensitive } \\
\text { equipment, } \\
\text { rolling stock }\end{array}$ & $\begin{array}{l}\text { e.g., Distribution } \\
\text { systems, ponds, reservoirs, } \\
\text { recreational water }\end{array}$ \\
\hline
\end{tabular}

Evaluate

400-2 citizen-performed decon options

Identify and assess consistency of decon options with regulatory requirements 


\section{DECONTAMINATION (expanded 403)}

Develop decontamination strategy

- ES\&H concerns

- Stakeholder concerns

- Decontamination reagents

- Delivery systems

Select decontamination methods including specific reagents and reagent delivery systems for media affected, considering for example:

Volume, Extent of contamination, Availability of resources, Accessibility, Weather

\begin{tabular}{|l|l|l|l|}
\hline \multicolumn{1}{|c|}{$\begin{array}{c}\text { Enclosedl } \\
\text { Semi-enclosed }\end{array}$} & \multicolumn{2}{|c|}{ Outdoor Areas } & Water \\
\hline $\begin{array}{l}\text { e.g., HVAC system, building } \\
\text { materials, fixed and moveable } \\
\text { property, sensitive equipment, } \\
\text { rolling stock }\end{array}$ & $\begin{array}{l}\text { Natural } \\
\text { e.g., soil, } \\
\text { vegetation, } \\
\text { agricultural } \\
\text { crops, } \\
\text { livestock }\end{array}$ & $\begin{array}{l}\text { Man-made } \\
\text { e.g., building } \\
\text { exteriors, } \\
\text { pavement } \\
\text { structures, fixed } \\
\text { and moveable } \\
\text { property, } \\
\text { sensitive } \\
\text { equipment, } \\
\text { rolling stock }\end{array}$ & $\begin{array}{l}\text { e.g., Distribution } \\
\text { systems, ponds, reservoirs, } \\
\text { recreational water }\end{array}$ \\
$\begin{array}{l}\text { Considering: } \\
\text { - Surface treatment }\end{array}$ & $\begin{array}{l}\text { Considering: } \\
\text { - Localized treatment } \\
\text { - Wide area treatment } \\
\text { - Wash down and treat }\end{array}$ & $\begin{array}{l}\text { Treatment } \\
\text { Nolumetric methods } \\
\text { monitor }\end{array}$ \\
\cline { 2 - 3 } & \multicolumn{2}{|c|}{} \\
\hline
\end{tabular}

Develop emergency response plans to address potential uncontrolled reagent release(s)

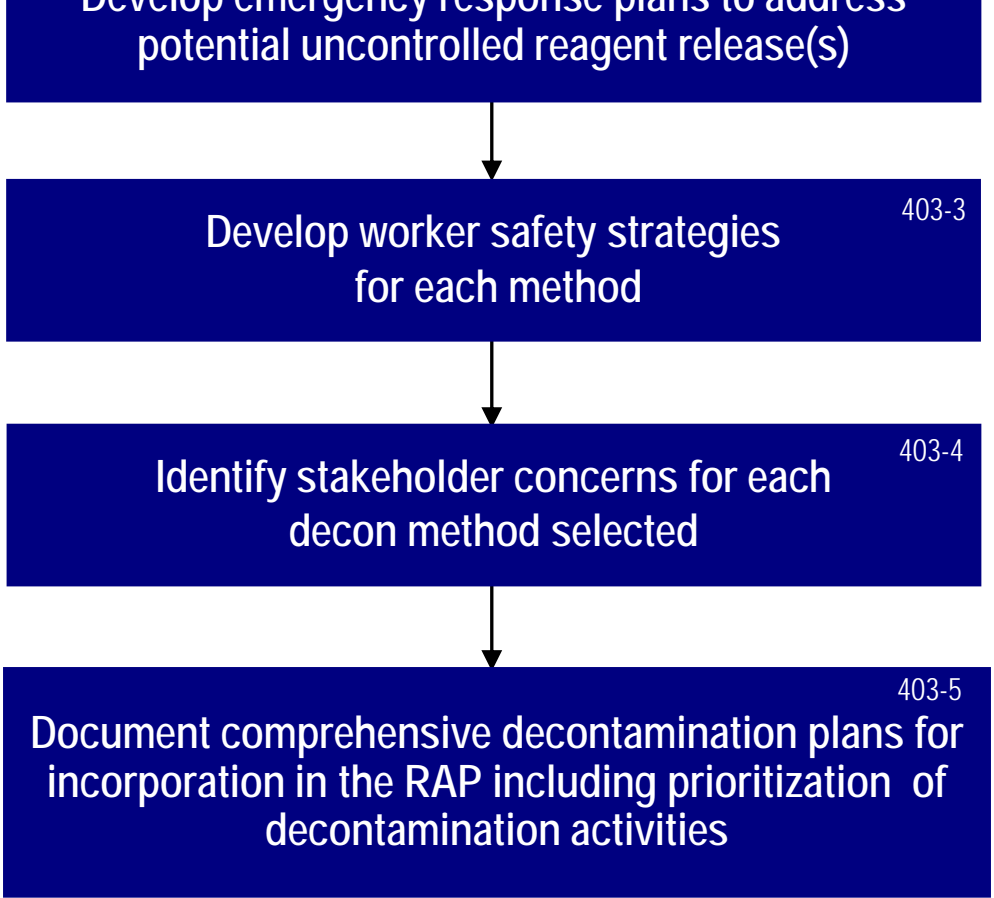

Identify stakeholder concerns for each decon method selected 
Develop appropriate decontamination strategy; determine packaging and transportation requirements

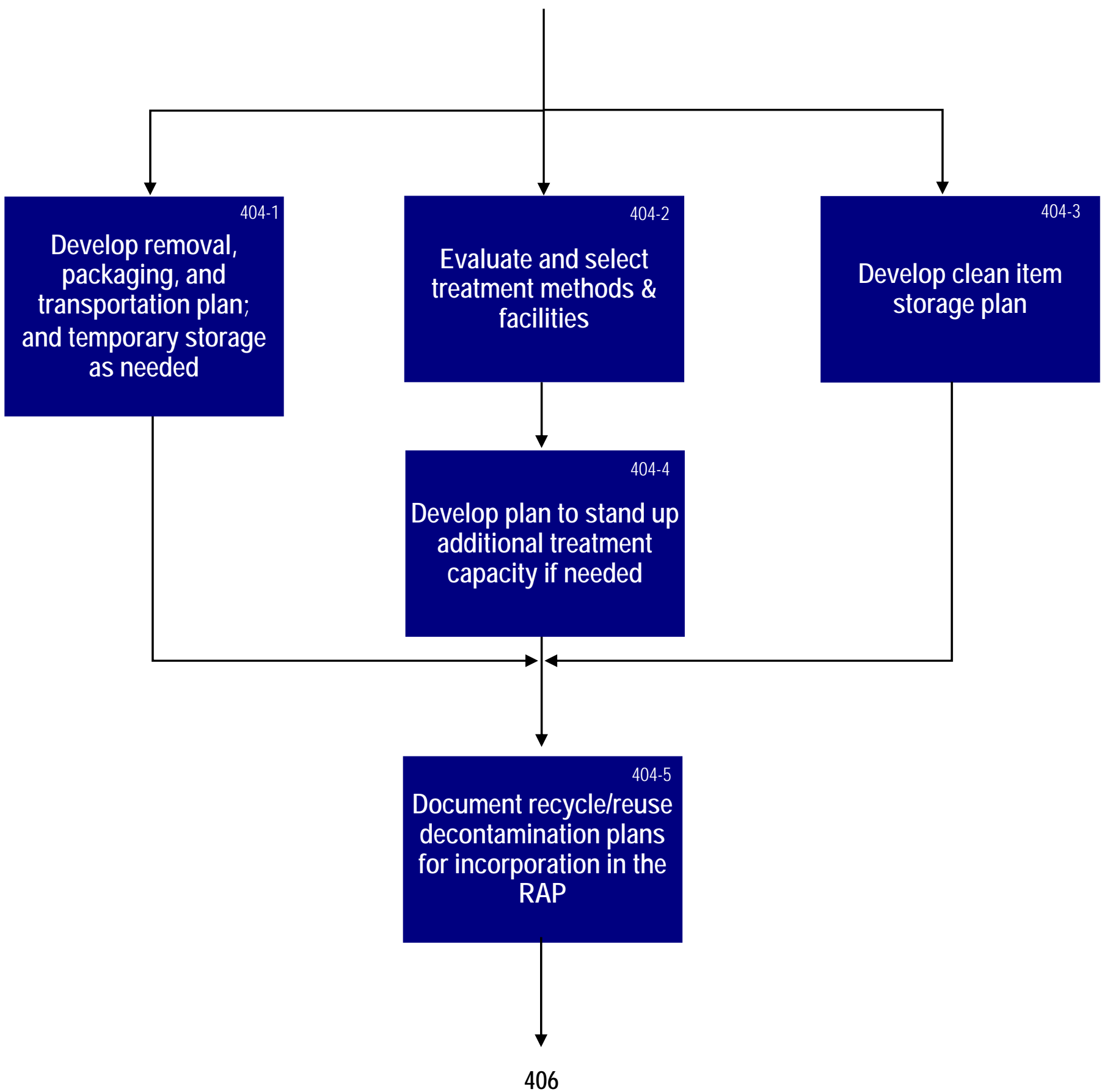


DECONTAMINATION

(expanded 405)
Select appropriate treatment and disposal site; determine packaging and transportation requirements

\section{5-1}

\section{Develop disposal strategy}

Select removal, packaging, and Evaluate and select treatment methods \& disposal facilities

transportation methods; and temporary storage solutions as needed

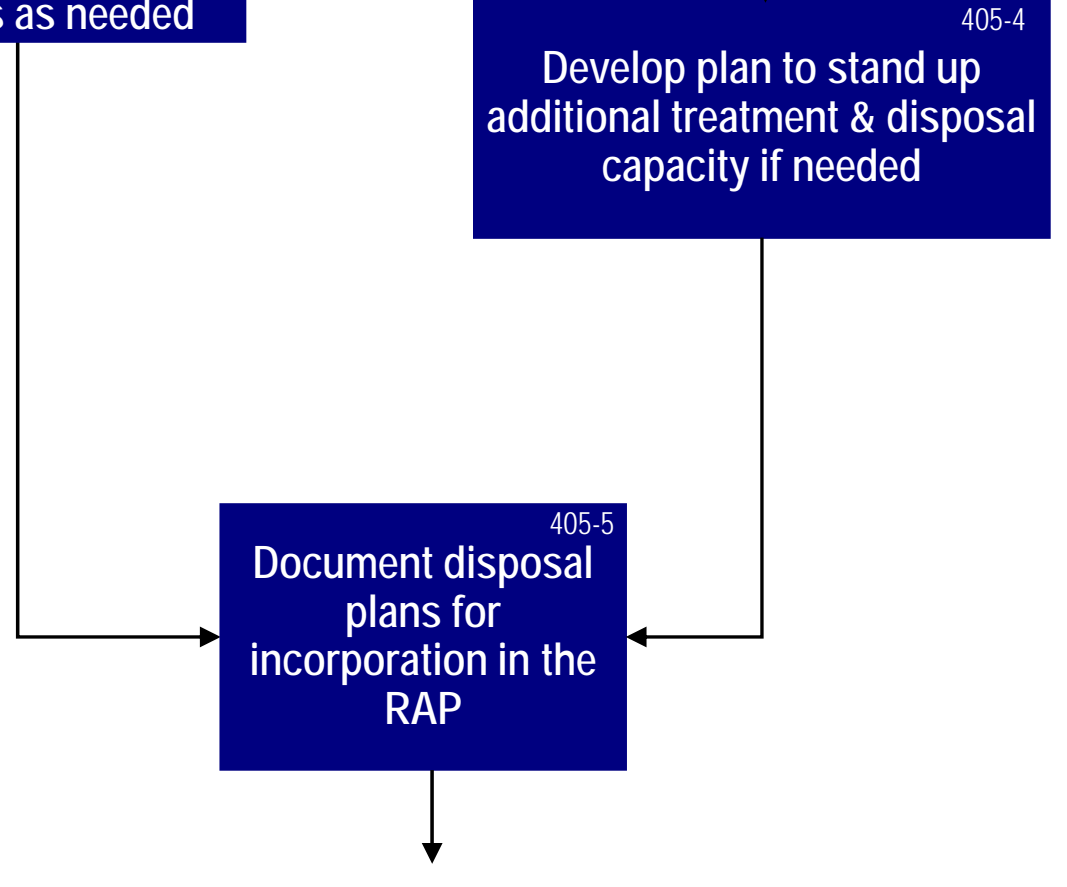




\section{DECONTAMINATION (expanded 406)}

Prepare remediation \& clearance sampling \& analysis action plan

- Select decontamination verification criteria

- Address clearance goals

Update prioritization of targets for decontamination

406-1<smiles>C1C[Te][Te]1</smiles>

Develop integrated decontamination strategy, considering:

- Priorities and optimization of resources

- Decontamination of outdoor areas before enclosed/semi-enclosed areas

Develop decon verification criteria and process criteria ( e.g., biological indicators, concentration, temperature, humidity, contact time, pH)<smiles>[Te]</smiles>

Develop clearance strategy, considering:

$406-4$

- Judgmental and statistical approaches

- Characterization approach and results

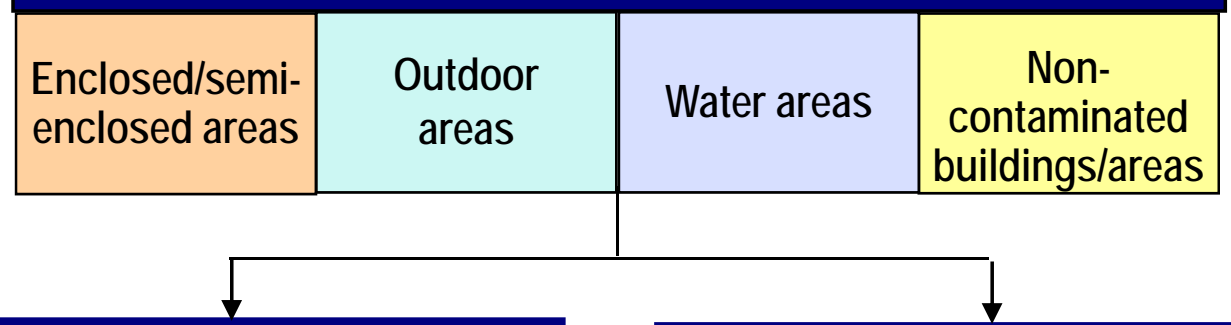

Develop a Remediation Action 406-5

Plan, include as necessary

- Ambient air monitoring plans

- Outdoor decon monitoring plans

Develop Clearance Sampling and Analysis Plan

Provide guidance for citizenperformed decontamination

Obtain EPA approvals or crisis exemptions

$\downarrow$

406-7

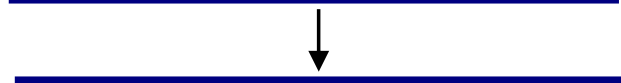




\section{CLEARANCE (expanded 500)}

Conduct clearance environmental sampling and final risk assessment as needed

Reassess need for clearance

in non-contaminated

potentially impacted areas

$500-2$

Reassess Clearance Sampling and Analysis Plan based on priorities, dependencies, and potential for recontamination

$500-3$

Execute Clearance Sampling and Analysis Plan

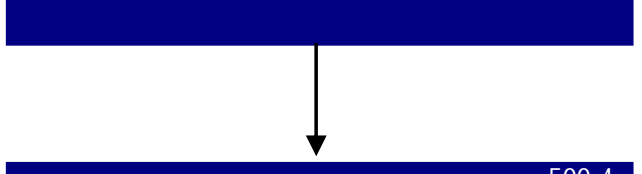

Conduct final technical risk assessment, considering results from clearance sampling
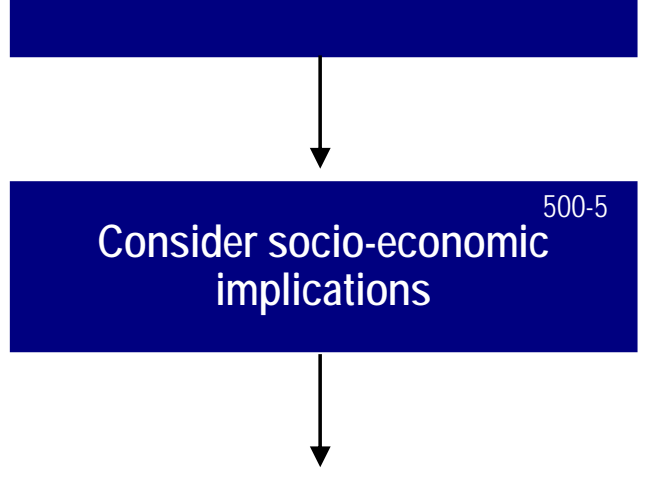

if needed

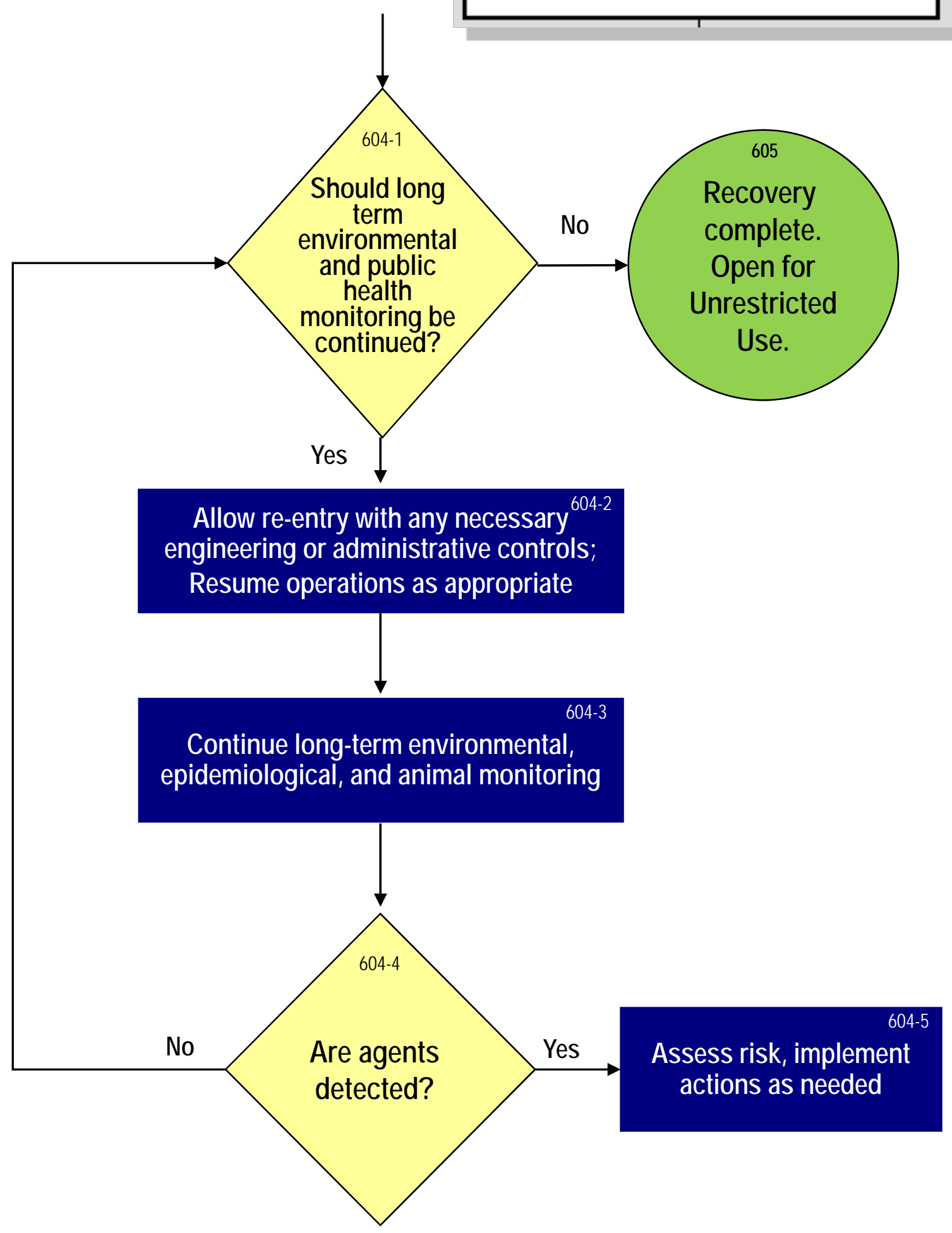

